\title{
Analysis and natural history of pituitary incidentalomas
}

\section{Syed Ali Imran', Churn-Ern Yip', Netee Papneja², Khaled Aldahmani ${ }^{3}$, Syed Mohammad', Fatima Imran', Deborah A Zwicker', Chris Theriault', Kara Thompson', David B Clarke ${ }^{5}$ and Stan Van Uum²}

${ }^{1}$ Division of Endocrinology and Metabolism, Dalhousie University, Halifax, Nova Scotia, Canada, ${ }^{2}$ Division of Endocrinology and Metabolism, Western University, London, Ontario, Canada, ${ }^{3}$ Department of Medicine, Tawam Hospital in affiliation with Johns Hopkins, Al Ain, UAE, ${ }^{4}$ Cape Breton Regional Hospital, Sydney, Nova Scotia, Canada, and ${ }^{5}$ Division of Neurosurgery, Dalhousie University, Halifax, Nova Scotia, Canada
Correspondence should be addressed to S A Imran

Email simran@dal.ca

\begin{abstract}
Objectives: Pituitary incidentalomas (PI) are frequently found on brain imaging. Despite their high prevalence, little is known about their long-term natural history and there are limited guidelines on how to monitor them.

Methods: We conducted a retrospective study to compare epidemiological characteristics at presentation and the natural history of $\mathrm{PI}$ in population-based vs referral-based registries from two tertiary-care referral centers in Canada. Results: A total of 328 patients with PI were included, of whom $73 \%$ had pituitary adenomas (PA) and $27 \%$ had non-pituitary sellar masses. The commonest indications for imaging were headache (28\%), dizziness (12\%) and stroke/transient ischemic attack (TIA) (9\%). There was a slight female preponderance $(52 \%)$ with a median age of 55 years at diagnosis; $71 \%$ presented as macroadenomas $(>10 \mathrm{~mm})$. Of PA, $25 \%$ were functioning tumors and at presentation $36 \%$ of patients had evidence of secondary hormonal deficiency (SHD). Of the total cohort, $68 \%$ were treated medically or conservatively whereas $32 \%$ required surgery. Most tumors ( $87 \%$ in non-surgery and $68 \%$ in post-surgery group) remained stable during follow-up. Similarly, $84 \%$ of patients in the non-surgery and $73 \%$ in the surgery group did not develop additional SHD during follow-up. The diagnosis of non-functioning adenoma was a risk factor for tumor enlargement and a change in SHD status was associated with a change in tumor size.

Conclusions: Our data suggest that most PI seen in tertiary-care referral centers present as macroadenomas and may frequently be functional, often requiring medical or surgical intervention.

\section{Introduction}

Pituitary incidentalomas (PI) are generally described as pituitary lesions without any overt features of pituitary disease that are found on brain imaging done for an unrelated indication (1). Although PI were initially described in 1936 (2), and are commonly encountered in clinical practice, little is known about the long-term natural history of PI. The prevalence of PI in autopsy series is reported to be around $3-27 \%(3,4,5)$ and around $10 \%$ on imaging studies (6). The available natural history data on PI are mostly derived from small-scale studies with relatively limited follow-up $(7,8,9)$. Consequently,

www.eje-online.org DOI: 10.1530/EJE-16-0041
() 2016 European Society of Endocrinology Printed in Great Britain the risk of developing hormonal dysfunction over longer follow-up is unknown, and it is unclear if this risk is affected by the size of the PI at initial discovery. It is also not known if there are any differences in epidemiological characteristics and natural history of PI in populationbased vs referral-based registries. Because of these unanswered questions, there are very limited guidelines on long-term management of PI. The aim of this study was to address these issues by assessing the natural history and epidemiological trend of a large cohort of patients with PI from two tertiary-care referral centers in Canada. 


\section{Patients and methods}

Data from two tertiary-care referral centers were analyzed for the study. The Halifax Neuropituitary (HNP) database, initially established in November 2005, is a comprehensive interlinked provincial registry that prospectively collects clinical, biochemical, radiological and surgical (when applicable) data on all neuropituitary patients within the province of Nova Scotia, which has a stable provincial population of almost 1 million (10). The pituitary database from the Western University in London, Ontario (Western), is a referral-based registry that prospectively collects data on all pituitary referrals to the tertiary-care center since January 2006. Inclusion criteria included: (i) PI defined as all sellar masses (SM) identified on brain imaging (MRI/CT) done for indications unrelated to pituitary disorders in patients who had no overt features of pituitary hormonal dysfunction or mass effect and (ii) had at least one complete clinical and biochemical assessment. All patients fulfilling these criteria, and who were seen between 1 January 2006 and 31 December 2014, were identified. Patients presenting with symptoms of SM such as visual field defects, overt symptoms of hypopituitarism or pituitary hormone excess were excluded. The study was approved by the Research Ethics Boards of the Capital Health and Western University. For the purpose of the study, we collected the following information: age, gender, diagnostic category of PI, indications of initial imaging, initial imaging modality, tumor size at presentation and during follow-up, length of follow-up, secondary hormonal deficiency (SHD) at presentation and during follow-up, management strategy, visual field, indications for surgery, pathology report (if applicable) and mortality.

\section{Diagnostic assessment for pituitary-related growths}

Pituitary-related growths are generally referred to as pituitary adenomas(PA). PAwere categorized based on their size into macroadenomas $(>10 \mathrm{~mm})$ or microadenomas $(<10 \mathrm{~mm})$ and functional status into either nonfunctioning adenomas (NFA) or functioning adenomas (FA). FA were further stratified based on the predominant hormonal release pattern. Prolactinomas (PRLoma) were defined as FA associated with detectable PA on imaging, a persistently elevated prolactin appropriate for the adenoma size, and presence of symptoms related to high prolactin. Growth hormone (GH) secreting adenoma was diagnosed on the basis of typical clinical features, an elevated age- and gender-matched serum insulin- like growth factor 1 (IGF1) and inability to suppress GH following a $75 \mathrm{~g}$ oral glucose load. Adrenocorticotropic hormone; adrenocorticotropin; corticotropin (ACTH) secreting adenomas were diagnosed based on clinical and biochemical features of hypercortisolism and evidence of pituitary origin of hypercortisolism based on a combination of the following tests: inappropriately normal or elevated ACTH, abnormal dexamethasone suppression test, adequate stimulation with corticotropin-releasing hormone test, inferior petrosal sinus sampling with or without a detectable pituitary tumor or positive tissue diagnosis. Thyroid stimulating hormone (TSH)-secreting adenoma was diagnosed based on an elevated free T4 (fT4), inappropriately normal or elevated TSH and presence of pituitary tumor and a positive tissue diagnosis. Non-functioning tumors (NFT) were diagnosed when there was no clinical and/ or biochemical evidence of hormonal over-secretion and in cases of macroadenoma where serum prolactin was less than $200 \mu \mathrm{g} / \mathrm{L}$ (normal range $=2.1-17.7 \mu \mathrm{g} / \mathrm{L}$ in males and $2.8-29.2 \mu \mathrm{g} / \mathrm{L}$ in females). For other sellar and parasellar masses, the diagnosis was based on typical clinical and radiological features; the latter was judged by an experienced neurosurgeon and/or directly obtained from the radiology report. For all patients who underwent surgery, tissue diagnosis was the primary method for making the diagnosis. PI without clinical and biochemical evidence of hormonal over-secretion despite positive immunohistochemistry staining were regarded as clinically silent and were categorized as NFT. Secondary hormone insufficiency was defined as follows: adrenal insufficiency was defined as either basal serum cortisol of $<130 \mathrm{nmol} / \mathrm{L}$ or failure of serum cortisol to rise $\geq 500 \mathrm{nmol} / \mathrm{L}$ after an insulin tolerance test or $250 \mu \mathrm{g}$ ACTH stimulation test based on our previously published data (11). Secondary hypothyroidism diagnosis was based on low fT4 with inappropriate normal or low TSH. Diabetes insipidus was diagnosed based on the presence of polyuria and polydipsia in addition to abnormal water deprivation test. We do not routinely perform dynamic testing for GH deficiency unless coverage for GH therapy is available; therefore, GH deficiency was defined as a low age- and gender-matched serum IGF1.

\section{Follow-up strategy}

All patients are routinely followed up for assessment. The standard follow-up protocol is to assess patients at 3 (in case if patients required immediate surgery) to 6 months (those who did not require immediate surgery) after 
initial clinic visit, every 12 months thereafter for 5 years and then every 12-24 months based on the physician's discretion. All patients underwent full pituitary hormonal assessment and sellar imaging with MRI or CT scan (in patients who were unable to undergo MRI) at the time of initial presentation and at each subsequent visit. For patients undergoing surgery, the data on indications for surgery were only available for those who were followed at the HNP clinic.

A significant increase in the size of PI was defined by an increase of $2 \mathrm{~mm}$ or more in any dimension. Newonset SHD was defined as loss of $\geq 1$ hormonal axis during follow-up. For patients who underwent surgery, the initial postoperative imaging size and SHD status were used as the new baseline to which subsequent imaging and SHD status was compared with in order to identify a significant change.

\section{Statistical analysis}

We used means and standard deviations to summarize continuous variables unless otherwise specified. Baseline characteristics between patients from the two hospital centers were compared using $\chi^{2}$ tests or Fisher's exact test where appropriate. Continuous variables were compared using Wilcoxon two-sample test or Student's $t$-test. Separate logistic regression models were used to identify baseline variables that were associated with surgery, an increase in SHD and a decrease in SHD. Change in SHD status was characterized with the use of Kaplan-Meier methods. Estimates of cumulative rates of being growth free at 1, 2 and 3 years were calculated. Patients were censored on date of death or date of last clinic visit.

\section{Results}

\section{Presentation of pituitary incidentalomas}

These are summarized in Table 1. Between 1 January 2006 and 31 December 2014, a total of 328 patients (220 from the HNP and 108 from Western) were diagnosed as PI. There was a slight female preponderance $(52 \%)$ with a median age at diagnosis of 55 years (interquartile range (IQR) 26), and a median size at diagnosis being $15 \mathrm{~mm}$ (IQR 11). The indications for initial imaging are summarized in Table 1. The most common indications overall for the initial diagnostic imaging were: headache (28\%), dizziness or vertigo (12\%), stroke or transient ischemic attack (9\%), head trauma (7\%), sinusitis (4\%), neck or facial pain (4\%), seizure $(2 \%)$, syncope $(2 \%)$, other $(28 \%)$, and missing or unknown (5\%). Other than a slightly higher percentage of headache and neck/facial pain in the Western cohort, there were no significant differences in the indications of initial imaging between the two centers.

The analysis of PI based on size at presentation is shown in Table 2. Most PI (71\%) were $\geq 10 \mathrm{~mm}$ in all categories, whereas $23 \%$ were $<10 \mathrm{~mm}$; the size was unknown in $6 \%$. When specifically looking at NFA based on initial size at presentation, $5 \%$ were $<5 \mathrm{~mm}, 13 \%$ were $5-9 \mathrm{~mm}, 75 \%$ were $>10 \mathrm{~mm}$, and the size was unknown in $7 \%$. Overall, MRI was the predominant initial imaging modality in HNP (60\%) compared with Western (39\%) (Table 1). The standardized incidence rate (SIR) for all PI was only based on the HNP cohort (as it pertains a defined population), which over the entire follow-up period was 1.63/100 000/year with a prevalence of $24.4 / 100$ 000/year.

\section{Diagnostic categories of $\mathrm{Pl}$ at presentation}

Overall, there were 239 (73\%) PA and 89 (27\%) nonpituitary SM with significantly more NFA and pituitary cysts found at Western whereas more Rathke's Cleft Cyst (RCC) and PRLoma found at HNP (Table 1). Of all PI $(n=328)$, the four most common SM were: NFA $(55 \%)$, RCC (14\%), PRLoma (11\%) and pituitary cysts (5\%). There were 60 predominantly FA subdivided into PRLoma (62\%), GH-producing adenoma (26\%) and ACTH adenoma (12\%). The 89 non-pituitary SM consisted of RCC (53\%), pituitary cyst (20\%), craniopharyngioma (17\%), meningioma (6\%), arachnoid cyst (2\%) and other lesions (2\%).

\section{Secondary hormonal deficiency at presentation}

There was no significant difference between the two centers in terms of either frequency or type of SHD (Table 1). The hormonal status at presentation was known in 252 patients, of whom 162 (64\%) did not have any hormonal deficiency, whereas $56(22 \%)$ had a single hormonal axis deficiency, 13 (5\%) had two hormonal axes deficiencies, $13(5 \%)$ had three hormonal axes deficiencies and 8 (3\%) had four or more hormonal axes deficiencies. The commonest SHD was hypogonadism (29\%) followed by hypothyroidism (13\%), GHD (12\%), adrenal insufficiency (6\%), and diabetes insipidus $(0.4 \%)$. When looking at only NFA and non-pituitary SM based on the initial size at presentation, the rate of SHD at presentation in those $<5 \mathrm{~mm}$ was $9 \%$ (1 out of 11 ), in $5-10 \mathrm{~mm}$ was $2.8 \%$ ( 1 out of 36 ) and those $>10 \mathrm{~mm}$ was $26 \%$ (34 out of 129 ). 
Table 1 Baseline characteristics of all pituitary incidentalomas at diagnosis.

\begin{tabular}{l}
\hline Variable \\
\hline Number of patients \\
F:M \\
Median age, years (IQR) \\
Reason for initial imaging \\
Headache \\
Dizziness or vertigo \\
Stroke/TIA \\
Trauma \\
Sinusitis \\
Neck or facial pain \\
Seizure \\
Syncope \\
Other \\
Missing or unknown \\
Size at diagnosis \\
Median size mm (IQR) \\
$n \geq 10$ mm \\
$n<10$ mm \\
Unknown \\
Initial imaging modality \\
MRI \\
CT \\
Type of SM \\
NFA \\
Rathke's cleft cysts \\
Prolactinomas \\
Pituitary cysts \\
GH producing adenomas \\
Craniopharyngiomas \\
ACTH producing adenomas \\
Meningioma \\
Arachnoid cysts \\
Other lesions \\
SHDothyroidism \\
Growth hormone \\
Adrenal insufficiency \\
Diabetes insipidus \\
Untus \\
\end{tabular}

\begin{tabular}{c}
\hline Halifax/CB \\
\hline 220 \\
$118: 102$ \\
$54(25.5)$
\end{tabular}

\begin{tabular}{c}
\hline Western \\
\hline 108 \\
$52: 56$ \\
$58.5(25)$
\end{tabular}

\begin{tabular}{c}
\hline Entire cohort \\
\hline 328 \\
$170: 158$ \\
$55(26)$
\end{tabular}

\begin{tabular}{c}
\hline P-value \\
\hline 0.34
\end{tabular}

$53(24 \%)$

$31(14 \%)$

$19(9 \%)$

$15(7 \%)$

$8(4 \%)$

$3(1 \%)$

$5(2 \%)$

$4(2 \%)$

$67(30 \%)$

$15(7 \%)$

15 (12)

$160(73 \%)$

$54(24 \%)$

$6(3 \%)$

$132(60 \%)$

$88(40 \%)$

$109(50 \%)$

$38(17 \%)$

$31(14 \%)$

7 (3\%)

$11(5 \%)$

$10(5 \%)$

$7(3 \%)$

$5(2 \%)$

$2(1 \%)$

0

$112(51 \%)$

$37(17 \%)$

$10(5 \%)$

$9(4 \%)$

5 (2\%)

$47(21 \%)$

$49(27 \%)$

$26(14 \%)$

$18(11 \%)$

$10(5 \%)$

$1(0.5 \%)$
$38(35 \%)$

$8(7 \%)$

$10(9 \%)$

$7(6 \%)$

$6(6 \%)$

$9(8 \%)$

$3(3 \%)$

$3(3 \%)$

$24(22 \%)$

0

$16(10.3)$

$73(68 \%)$

$22(20 \%)$

$13(12 \%)$

$39(36 \%)$

$69(64 \%)$

$70(65 \%)$

$9(8 \%)$

$6(6 \%)$

$11(10 \%)$

5 (5\%)

$5(5 \%)$

0

0

0

$2(2 \%)$

$50(46 \%)$

$19(18 \%)$

$3(3 \%)$

$4(4 \%)$

$3(3 \%)$

$29(27 \%)$

$26(33 \%)$

$7(9 \%)$

$11(14 \%)$

5 (6\%)

0
$91(28 \%)$

$39(12 \%)$

$29(9 \%)$

$22(7 \%)$

$14(4 \%)$

$12(4 \%)$

$8(2 \%)$

$7(2 \%)$

$91(28 \%)$

$15(5 \%)$

15 (11)

$233(71 \%)$
$76(23 \%)$

$19(6 \%)$

$171(52 \%)$

$157(48 \%)$

$179(55 \%)$

$47(14 \%)$

$37(11 \%)$

$18(5 \%)$

$16(5 \%)$

$15(5 \%)$

$7(2 \%)$

$5(2 \%)$

$2(0.5 \%)$

$2(0.5 \%)$

$162(49 \%)$

$56(17 \%)$

$13(4 \%)$

$13(4 \%)$

$8(2 \%)$

$76(23 \%)$

75 (29\%)

$33(13 \%)$

$29(12 \%)$

$15(6 \%)$

$1(0.4 \%)$
0.0349

0.0788

0.8519

0.9088

0.4013

0.0030 *

0.7214 *

0.6880 *

0.1176

0.0034 *

0.69

$<0.0001$

0.0091

0.0299

0.0217

0.0089

0.8837

1.000 *

0.1004 *

0.1758

1.000 *

0.1077 *

$0.8731^{\S}$

0.3527

0.2427

0.4648

0.7761 * $1.000 *$

*Fishers exact test; ${ }^{\S}$ Wilcoxon two-sample test.

\section{Course during follow-up and management strategies}

The follow-up scheme is summarized in Fig. 1. Of the 328 patients with a median follow-up time of 3.02 years (IQR $4.02), 68 \%$ were either monitored or treated with medical therapy, whereas $32 \%$ underwent surgical resection. We assessed which factors at presentation increase the chance for undergoing surgery. Logistic regression models showed increased odds ratio were found for presence of FA(OR: 23.81 (95\%CI: 5.45-103.94) P<0.0001), SHD at presentation (OR: 4.69 (95\% CI: 2.54-8.63) $P \leq 0.001)$ and larger size at presentation (OR: 1.234 (95\% CI: $1.148-1.328)$ per each $2 \mathrm{~mm}$ increase in initial size) $(P<0.0001)$. For those who underwent surgery, 35\% had surgery in $\leq 90$ days of diagnosis whereas $65 \%$ had surgery after 90 days. Sixteen patients (15\%) required a 
Table 2 Sellar mass (SM) by size at presentation.

\begin{tabular}{|c|c|c|c|c|}
\hline Type of SM & $\begin{array}{l}\geq \mathbf{1 0} \mathbf{~ m m} \\
(n=233)\end{array}$ & $\begin{array}{c}<\mathbf{1 0} \mathbf{~ m m} \\
(n=76) \\
\end{array}$ & $\begin{array}{c}\text { Unknown } \\
(n=19) \\
\end{array}$ & $\begin{array}{c}\text { Total } \\
(n=328)\end{array}$ \\
\hline NFA & $134(75 \%)$ & $33(18 \%)$ & $12(7 \%)$ & 179 \\
\hline Prolactinoma & $28(76 \%)$ & $4(11 \%)$ & $5(13 \%)$ & 37 \\
\hline $\begin{array}{l}\text { GH producing } \\
\text { adenoma }\end{array}$ & $12(75 \%)$ & $4(25 \%)$ & 0 & 16 \\
\hline $\begin{array}{l}\text { ACTH producing } \\
\text { adenoma }\end{array}$ & $6(86 \%)$ & $1(14 \%)$ & 0 & 7 \\
\hline RCC & $26(55 \%)$ & $21(45 \%)$ & 0 & 47 \\
\hline Pituitary cyst & $10(55 \%)$ & $8(45 \%)$ & 0 & 18 \\
\hline Craniopharyngioma & $9(60 \%)$ & $4(27 \%)$ & $2(13 \%)$ & 15 \\
\hline Meningioma & $5(100 \%)$ & 0 & 0 & 5 \\
\hline Arachnoid cyst & $2(100 \%)$ & 0 & 0 & 2 \\
\hline Others & $1(50 \%)$ & $1(50 \%)$ & 0 & 2 \\
\hline
\end{tabular}

second surgery of which 50\% had enlarging NFA, 31\% had GH adenoma, 13\% had ACTH adenoma and 6\% had a pituitary cyst. There were no significant differences between the two centers (data not shown). Indications for initial surgery were only available in the HNP surgery cohort $(n=74)$, which included: impaired vision $(27 \%)$, growth of the tumor in follow-up (20\%), FA (19\%), contact with optic chiasm (8\%), diagnostic resection (3\%) and patient preference $(1 \%)$ whereas in $22 \%$ patients it was not recorded.

\section{Tumor size on follow-up}

Of the initial 328 patients, 265 had at least one or more follow-up imaging study. Of those 265 patients, 202 (76\%) were either monitored or treated medically, whereas 63
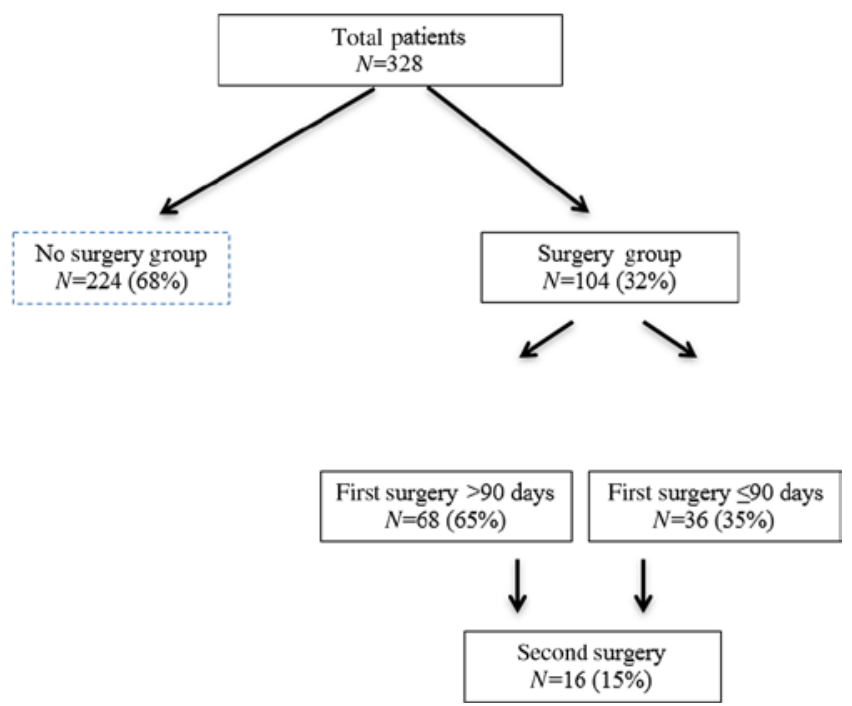

\section{Figure 1}

Flow diagram of cohort upon follow-up.
(24\%) underwent surgical resection with a median duration of follow-up of 2.81 years (IQR: 3.5 ) and 5.3 years (IQR: 4.33) respectively. None of the patients with NFA were treated with dopamine agonists or other anti-tumor treatments. Of the 202 patients not undergoing surgery initially, $87 \%$ had no significant change in the size of their PI, $1 \%$ had a significant decrease in size whereas $12 \%$ had a significant increase. Comparing NFA with non-pituitary SM, NFA had a higher risk of enlargement with an OR of 3.92 (95\% CI: 1.27-12.07) $(P=0.017)$ (Table 3). Age at presentation and gender did not impact risk for tumor growth.

On follow-up of the 63 patients who underwent surgery, $68 \%$ had no significant change in the size of the residual post-surgery tissue, whereas $10 \%$ had a significant decrease in size and $22 \%$ had a significant increase. Because of the small sample size, there was low power to detect any significant risk factor associated with tumor enlargement after surgery. However, there was a trend for subsequent tumor growth for every $2 \mathrm{~mm}$ increase in the initial post-surgery residual tumor size $(\mathrm{OR}=1.156(95 \%$ CI: 0.988-1.352) $P=0.053$ ).

We analyzed our data to determine whether tumor size at presentation is predictive of further growth in patients with NFA and those with non-pituitary SM only $(n=133)$. Of those $<5 \mathrm{~mm}$ in size at initial presentation $(n=7)$ none enlarged during follow-up; in those between 5 and $10 \mathrm{~mm}$ at presentation $(n=22)$, subsequent enlargement was seen in $14 \%$; and in those $>10 \mathrm{~mm}$ at presentation $(n=104)$, there was an enlargement of tumors in $25 \%$ patients $(P=0.06)$. For NFA and non-pituitary SM that did not require surgery, the estimated rate of tumor stability (without growth) at 1 year was 98\% (95\% CI: 93-99), at 2 years was 95\% (95\% CI: 88-98) and at 3 years was 91\% (95\% CI: 80-96).

There were 11 deaths during follow-up. The cause of death was identified in six patients and included cerebrovascular disease in two patients, and one each of metastatic urothelial cancer, post cardiac catheterization, esophageal adenocarcinoma and aspiration pneumonia. Pituitary lesions had been stable in all patients before death except in one case where the pituitary lesion had enlarged during the 6 months before death. New-onset VF defects were not identified in any patient during follow-up.

\section{Hormonal deficiency during follow-up}

Of the initial 328 patients, 229 had at least one follow-up pituitary hormonal evaluation with an overall median duration of follow-up of 3.75 years (IQR: 4.33). Of those, 169 (74\%) were monitored or treated with medical therapy 
Table 3 Size change of SM in follow-up as indicated by HNP classification $(n=265)$.

\begin{tabular}{|c|c|c|c|c|c|}
\hline Cohort & Type of SM & No change & Decrease & Increase & Total \\
\hline \multicolumn{6}{|c|}{ No surgery } \\
\hline & NFA & $79(80 \%)$ & 0 & $20(20 \%)$ & 99 \\
\hline & Prolactinoma & $32(94 \%)$ & $1(3 \%)$ & $1(3 \%)$ & 34 \\
\hline & GH producing adenoma & $2(100 \%)$ & 0 & 0 & 2 \\
\hline & Cushing's disease & 0 & 0 & 0 & 0 \\
\hline & Rathke's cleft cyst & $34(97 \%)$ & 0 & $1(3 \%)$ & 35 \\
\hline & Pituitary cyst & $14(88 \%)$ & 0 & $2(12 \%)$ & 16 \\
\hline & Craniopharyngioma & $10(91 \%)$ & 0 & $1(9 \%)$ & 11 \\
\hline & Meningioma & $2(67 \%)$ & $1(33 \%)$ & 0 & 3 \\
\hline & Arachnoid cyst & $2(100 \%)$ & 0 & 0 & 2 \\
\hline & Total & $175(87 \%)$ & $2(1 \%)$ & $25(12 \%)$ & 202 \\
\hline \multicolumn{6}{|l|}{ Surgery } \\
\hline & NFA & $30(71 \%)$ & $2(5 \%)$ & $10(24 \%)$ & 42 \\
\hline & Prolactinoma & 0 & $1(100 \%)$ & 0 & 1 \\
\hline & GH producing adenoma & $5(63 \%)$ & $2(25 \%)$ & $1(12 \%)$ & 8 \\
\hline & Cushing's disease & $3(75 \%)$ & 0 & $1(25 \%)$ & 4 \\
\hline & Rathke's cleft cyst & $2(40 \%)$ & $1(20 \%)$ & $2(40 \%)$ & 5 \\
\hline & Pituitary cyst & 0 & 0 & 0 & 0 \\
\hline & Craniopharyngioma & $2(100 \%)$ & 0 & 0 & 2 \\
\hline & Meningioma & $1(100 \%)$ & 0 & 0 & 1 \\
\hline & Total & $43(68 \%)$ & $6(10 \%)$ & $14(22 \%)$ & 63 \\
\hline
\end{tabular}

and $60(26 \%)$ underwent surgery with a median duration of follow-up of 3.12 years (IQR: 3.88 ) and 4.82 years (IQR: 4.12) respectively.

Of the 169 non-surgery patients, 142 (84\%) had no change in SHD status, 9 (5\%) had an improvement in SHD status, 15 (9\%) patients developed additional SHD and 3 (2\%) had a fluctuating change in SHD (Table 4). As expected, improvement in SHD was seen mostly in PRLoma (7 out of 9) along with one each of craniopharyngioma and NFA. Improvement in SHD was not associated with age at diagnosis or initial size at presentation. Fifteen (9\%) patients developed additional SHD, of whom three

Table 4 Change in SHD status during follow-up $(n=229)$.

\begin{tabular}{|c|c|c|c|c|c|c|}
\hline \multirow[b]{2}{*}{ Cohort } & \multirow[b]{2}{*}{ Type of SM } & \multicolumn{4}{|c|}{ SHD status during follow-up } & \multirow[b]{2}{*}{ Total } \\
\hline & & No change & Worse & Improved & Fluctuating & \\
\hline \multicolumn{7}{|c|}{ No surgery } \\
\hline & NFA & $68(88 \%)$ & $1(1 \%)$ & $8(11 \%)$ & 0 & 77 \\
\hline & Prolactinoma & $22(65 \%)$ & $7(21 \%)$ & $4(11 \%)$ & $1(3 \%)$ & 34 \\
\hline & GH producing adenoma & $2(100 \%)$ & 0 & 0 & 0 & 2 \\
\hline & Cushing's disease & 0 & 0 & 0 & 0 & 0 \\
\hline & Rathke's cleft cyst & $29(97 \%)$ & 0 & 0 & $1(3 \%)$ & 30 \\
\hline & Pituitary cyst & $11(100 \%)$ & 0 & 0 & 0 & 11 \\
\hline & Craniopharyngioma & $6(60 \%)$ & $1(10 \%)$ & $3(30 \%)$ & 0 & 10 \\
\hline & Meningioma & $2(67 \%)$ & 0 & 0 & $1(33 \%)$ & 3 \\
\hline & Arachnoid cyst & $2(100 \%)$ & 0 & 0 & 0 & 2 \\
\hline & Total & $142(84 \%)$ & $9(5 \%)$ & $15(9 \%)$ & $3(2 \%)$ & 169 \\
\hline \multicolumn{7}{|l|}{ Surgery } \\
\hline & NFA & $27(71 \%)$ & $1(3 \%)$ & $8(21 \%)$ & $2(5 \%)$ & 38 \\
\hline & Prolactinoma & 0 & $1(100 \%)$ & 0 & 0 & 1 \\
\hline & GH producing adenoma & $6(86 \%)$ & 0 & $1(14 \%)$ & 0 & 7 \\
\hline & Cushing's disease & $3(75 \%)$ & $1(25 \%)$ & 0 & 0 & 4 \\
\hline & Rathke's cleft cyst & $4(80 \%)$ & $1(20 \%)$ & 0 & 0 & 5 \\
\hline & Pituitary cyst & 0 & 0 & 0 & 0 & 0 \\
\hline & Craniopharyngioma & $2(100 \%)$ & 0 & 0 & 0 & 2 \\
\hline & Meningioma & $1(50 \%)$ & $1(50 \%)$ & 0 & 0 & 2 \\
\hline & Other & $1(100 \%)$ & 0 & 0 & 0 & 1 \\
\hline & Total & $44(74 \%)$ & $5(8 \%)$ & $9(15 \%)$ & $2(3 \%)$ & 60 \\
\hline
\end{tabular}


showed tumor enlargement whereas others remained unchanged. The change in SHD status occurred within a median time of 8.4 months of a change in tumor volume.

Of the 60 patients who underwent surgery, 44 (73\%) had no change in SHD, 5 (8\%) had an improvement in SHD status, 9 (15\%) developed additional SHD and 2 (3\%) had fluctuating SHD (Table 4). The worsening SHD was associated with a change in the tumor size within 1 month of the change in SHD status. No other factors were associated with worsening of SHD.

When specifically looking at NFA overall $(n=111)$ with initial size categories of $<5 \mathrm{~mm}, 5-10 \mathrm{~mm}$, and $>10 \mathrm{~mm}$, there was no statistically significant difference in progression of SHD status over time based on these initial size categories.

When looking at NFA and non-pituitary SM who did not require surgery, the SHD status remained mostly unchanged with an estimated stability of pituitary hormonal function at 1 year being 98\% (95\% CI: 93-99), at 2 years being 97\% (95\% CI: 91-99) and at 3 years being 93\% (95\% CI: 85-97).

\section{Discussion}

Despite the high prevalence of PI, most data regarding their natural history are relatively limited and are primarily derived from smaller studies (12). In this study we assessed the natural history of PI in a large population from two tertiary-care centers in Canada. Furthermore, our study also allowed us to compare data from a comprehensive provincial registry (HNP) and a referralbased registry (Western) to identify different trends. There were some differences between the two main registries; most significantly in the initial imaging modality, where MRI was the commonest primary modality in the HNP as opposed to CT in the Western. This could partly be due to the differences in the availability of MRI imaging facilities, referral patterns from other specialists and local preferences. Nonetheless, the population of patients from both registries was overall very similar.

Based on our comprehensive registry data from HNP, the population prevalence of PI was 24.4/100 000/year and the SIR was 1.63/100 000/year. To our knowledge, this is the first study that has systematically reported the population prevalence of PI. However, it is noteworthy that PI were identified on brain MRI or CT, which may underestimate their true prevalence. Unlike previous studies that reported an overall female preponderance (12), our data showed no significant gender difference in the prevalence of PI. Our study also showed a clear preponderance of macroadenomas at presentation $(71 \%)$. This trend was previously shown in other studies as well (13). However, more interestingly, PI across all categories in our cohort were more likely to present as macroadenomas. On the contrary, the autopsy studies predominantly showed microadenomas (14). Possible explanations of this trend may be that most PI were detected on MRI and CT scans of the brain rather than that of the sella which may have missed smaller lesions. There is also a possibility that the radiologists may have under-reported small lesions, since another recent study reported a slightly higher proportion (37.5\%) of microadenomas (15).

The most common indications for initial imaging were: headache (28\%), dizziness/vertigo (12\%), stroke/ TIA (9\%) and trauma (7\%). Not only were they consistent between the two centers, but they were also similar to a large series reported from Japan (16). The association of headaches with PI remains a controversial issue. Headache as an associated symptom has been described in small and large as well as functioning and nonfunctioning pituitary tumors $(17,18)$. Headaches have been reported to be the most common reason for requesting CT scans of the brain in the ambulatory care setting in Ontario, Canada, accounting for $27 \%$ of outpatient CT scans (19). This correlates well with our finding that it accounted for $28 \%$ of the indications for initial imaging. Furthermore, resolution of headache in some patients after surgery has been reported but it is unclear if it is due to the removal of the tumor, effect of anesthesia or the natural history of headaches $(20,21)$. This raises an important question: if there were a clear causal association between headache and PI, would it be correct to label these lesions as incidental? Although other published studies to date have labeled pituitary adenomas that are identified during workup of headache as PI, this remains to be clarified in future studies.

As previously reported in other studies $(9,22)$, the commonest lesion in our cohort was NFA, which constituted around 55\% of the overall group whereas PRLoma constituted the predominant functioning PA. The overall preponderance of NFA makes intuitive sense because NFA are clinically silent until they present with features associated with compression of the adjacent tissue. Similarly, the higher preponderance of RCC in our study is also consistent with other large series (16).

The risk of hormonal deficiency in PI is significant. Among patients in whom a complete endocrine assessment was conducted at presentation, 36\% had evidence of SHD. Data regarding SHD are quite variable in the sense that a large series of 506 patients from Japan 
did not report SHD (16) whereas a smaller study of 61 patients from Greece showed SHD in 61\% patients (13). Our data show a high risk of secondary hypogonadism and hypothyroidism in PI. Therefore, complete endocrine assessment at the time of presentation in all PI is crucial. More importantly, to our knowledge this is the first study to assess the SHD status in association with changes in tumor size. Our data showed that most patients (84 and $74 \%$ in surgery and non-surgery groups respectively) had no change in their SHD status during follow-up. PRLoma were significantly more likely to have an improvement in SHD and tumor size. This would be expected given PRLoma treatment with dopamine agonists is very effective and would cause tumor shrinkage in addition to improvement in prolactin and SHD associated with it. Apart from an association with change in tumor size we found no other risk factors associated with developing of additional SHD. Data regarding long-term trends in tumor size change are sparse. Donovan and coworkers followed 31 patients with PI for 6 years and showed tumor enlargement in 3 (10\%) and shrinkage in 4 (13\%) cases (7). Similarly, the study from Japan (16) reported enlargement in $12.4 \%$ and shrinkage in $12 \%$ PI over a mean period of 26.9 months. Our data showed tumor enlargement in $15 \%$ and shrinkage in $3 \%$ whereas $82 \%$ PI remained stable over a mean period of 3.27 years. Our data also suggest that over $90 \%$ patients with NFA and non-pituitary SM that did not undergo surgery do not enlarge or develop additional SHD over 3 years. Therefore, such patients may not require rigorous follow-up; however, their long-term stability remains to be studied.

There has been an interest in the possibility that very small micro NFA have a different natural history from that of larger micro NFA and macro NFA. We attempted to assess the change in tumor size and SHD based on the initial size of the NFA categorized by $<5 \mathrm{~mm}, 5-9 \mathrm{~mm}$ and $\geq 10 \mathrm{~mm}$. Overall, our study did not show evidence for a significant difference in the natural history of NFA initially found $<5 \mathrm{~mm}, 5-9 \mathrm{~mm}$ and $\geq 10 \mathrm{~mm}$, thus suggesting that patients with small NFA will require consistent follow-up. Larger studies are required to confirm this observation further.

Our study has several strengths. We have analyzed data from a referral-based center as well as a populationbased registry and striking similarities in the trends between two centers indicate that PI have a fairly consistent pattern. Apart from one previous study (16), this is the largest series of PI and the largest data published from North America. Furthermore, this is the first study to look at the incidence and prevalence of PI in a population-based registry. However, there are limitations as well. Some of the data were collected retrospectively and information on pituitary function, tumor size and radiological characteristics of all patients were not available. Future studies should compare whether PI's natural history is similar to those SM that are not discovered incidentally and the possible impact on follow-up management.

In summary, our data, which constitutes the largest series of PI from North America, showed that most PI present as macroadenomas and require comprehensive hormonal testing initially and over time. Furthermore, a considerable proportion of PI require immediate surgery whereas those who do not require surgery tend to have a benign course with minimal risk of tumor enlargement or developing additional hormonal deficiencies.

Declaration of interest

The authors declare that there is no conflict of interest that could be perceived as prejudicing the impartiality of the research reported.

\section{Funding}

This research did not receive any specific grant from any funding agency in the public, commercial or not-for-profit sector.

\section{References}

1 Freda PU, Beckers AM, Katznelson L, Molitch ME, Montori VM, Post KD \& Vance ML. Pituitary incidentaloma: an endocrine society clinical practice guideline. Journal of Clinical Endocrinology \& Metabolism 201196 894-904. (doi:10.1210/jc.2010-1048)

2 Costello RT. Subclinical adenoma of the pituitary gland. American Journal of Pathology 193612205.

3 Chacko AG \& Chandy MJ. Incidental pituitary macroadenomas. British Journal of Neurosurgery 19926 233-236. (doi:10.3109/02688699209002931)

4 Reincke M, Allolio B, Saeger W, Menzel J \& Winkelmann W. The 'incidentaloma' of the pituitary gland. Is neurosurgery required? JAMA $19902632772-2776$.

5 Teramoto A, Hirakawa K, Sanno N \& Osamura Y. Incidental pituitary lesions in 1,000 unselected autopsy specimens. Radiology 1994193 161-164. (doi:10.1148/radiology.193.1.8090885)

6 Hall WA, Luciano MG, Doppman JL, Patronas NJ \& Oldfield EH. Pituitary magnetic resonance imaging in normal human volunteers: occult adenomas in the general population. Annals of Internal Medicine 1994120 817-820. (doi:10.7326/0003-4819-120-10-19940515000001)

7 Donovan LE \& Corenblum B. The natural history of the pituitary incidentaloma. Archives of Internal Medicine 1995155 181-183. (doi:10.1001/archinte.1995.00430020067008)

8 Arita K, Tominaga A, Sugiyama K, Eguchi K, Iida K, Sumida M, Migita K \& Kurisu K. Natural course of incidentally found nonfunctioning pituitary adenoma, with special reference to pituitary apoplexy during follow-up examination. Journal of Neurosurgery 2006 104 884-891. (doi:10.3171/jns.2006.104.6.884) 
9 Day PF, Guitelman M, Artese R, Fiszledjer L, Chervin A, Vitale NM, Stalldecker G, Miguel VD, Cornalo D, Alfieri A et al. Retrospective multicentric study of pituitary incidentalomas. Pituitary 20057 145-148.

10 Al-Dahmani K, Mohammad S, Imran F, Theriault C, Doucette S, Zwicker D, Yip CE, Clarke DB \& Imran SA. Sellar masses: an epidemiological study. Canadian Journal of Neurological Sciences 2016 43 291-297. (doi:10.1017/cjn.2015.301)

11 Yip CE, Stewart SA, Imran F, Clarke DB, Mokashi A, Kaiser SM \& Imran SA. The role of morning basal serum cortisol in assessment of hypothalamic pituitary-adrenal axis. Clinical and Investigative Medicine. Médecine Clinique et Experimentale 201336 E216-E222.

12 Fernandez-Balsells MM, Murad MH, Barwise A, Gallegos-Orozco JF, Paul A, Lane MA, Lampropulos JF, Natividad I, Perestelo-Perez L, Ponce de Leon-Lovaton PG et al. Natural history of nonfunctioning pituitary adenomas and incidentalomas: a systematic review and metaanalysis. Journal of Clinical Endocrinology \& Metabolism 201196 905-912.

13 Anagnostis P, Adamidou F, Polyzos SA, Efstathiadou Z, Panagiotou A \& Kita M. Pituitary incidentalomas: a single-centre experience. International Journal of Clinical Practice 201165 172-177. (doi:10.1111/ijcp.2011.65.issue-2)

14 Orija IB, Weil RJ \& Hamrahian AH. Pituitary incidentaloma. Best Practice \& Research Clinical Endocrinology \& Metabolism 201226 47-68.

15 Esteves C, Neves C, Augusto L, Menezes J, Pereira J, Bernardes I, Fonseca J \& Carvalho D. Pituitary incidentalomas: analysis of a neuroradiological cohort. Pituitary 201518 777-781. (doi:10.1007/ s11102-015-0652-7)
16 Sanno N, Oyama K, Tahara S, Teramoto A \& Kato Y. A survey of pituitary incidentaloma in Japan. European Journal of Endocrinology 2003149 123-127. (doi:10.1530/eje.0.1490123)

17 Levy MJ, Jäger HR, Powell M, Matharu MS, Meeran K \& Goadsby PJ. Pituitary volume and headache: size is not everything. Archives of Neurology 200461 721-725. (doi:10.1001/archneur.61.5.721)

18 Abe T, Matsumoto K, Kuwazawa J, Toyoda I \& Sasaki K. Headache associated with pituitary adenomas. Headache 199838 782-786. (doi:10.1046/j.1526-4610.1998.3810782.x)

19 You JJ, Gladstone J, Symons S, Rotstein D, Laupacis A \& Bell CM. Patterns of care and outcomes after computed tomography scans for headache. American Journal of Medicine 2011124 58-63. (doi:10.1016/j.amjmed.2010.08.010)

20 Levy MJ, Matharu MS, Meeran K, Powell M \& Goadsby PJ. The clinical characteristics of headache in patients with pituitary tumours. Brain: A Journal of Neurology 2005128 1921-1930.

21 Wolf A, Goncalves S, Salehi F, Bird J, Cooper P, Van Uum S, Lee DH, Rotenberg BW \& Duggal N. Quantitative evaluation of headache severity before and after endoscopic transsphenoidal surgery for pituitary adenoma. Journal of Neurosurgery 2015 In press. (doi:10.3171/2015.5.JNS1576)

22 Feldkamp J, Santen R, Harms E, Aulich A, Modder U \& Scherbaum WA. Incidentally discovered pituitary lesions: high frequency of macroadenomas and hormone-secreting adenomasresults of a prospective study. Clinical Endocrinology 199951 109-113. (doi:10.1046/j.1365-2265.1999.00748.x)

Received 14 January 2016

Revised version received 30 March 2016

Accepted 1 April 2016 